\title{
Use of vitamin D drops leading to kidney failure in a 54-year-old man
}

\author{
Bourne L. Auguste MD, Carmen Avila-Casado MD PhD, Joanne M. Bargman MD
}

Cite as: CMAJ 2019 April 8;191:E390-4. doi: 10.1503/cmaj.180465

A 54-year-old man was referred urgently to the nephrology clinic by his family physician for suspected acute kidney injury, with a creatinine level of $376 \mu \mathrm{mol} / \mathrm{L}$. He had recently returned from a trip to Southeast Asia, where he had spent extensive periods sunbathing $(6-8 \mathrm{~h} / \mathrm{d})$ for 2 weeks. His medical history included hypertension, dyslipidemia and gout, for which he was taking perindopril $8 \mathrm{mg}$ daily, rosuvastatin $10 \mathrm{mg}$ daily, amlodipine $10 \mathrm{mg}$ daily, indapamide $2.5 \mathrm{mg}$ daily and febuxostat $80 \mathrm{mg}$ daily.

On his return to Canada, the patient's creatinine level had initially increased from his baseline of $100 \mu \mathrm{mol} / \mathrm{L}$ to $132 \mu \mathrm{mol} / \mathrm{L}$. His family physician instructed him to discontinue his antihypertensive and diuretic agents temporarily on the premise that he had possible extracellular fluid depletion from the heat exposure. Despite this measure, on repeat measurement 4 weeks later, the patient's creatinine level had risen to $376 \mu \mathrm{mol} / \mathrm{L}$. During this 4-week period, he had not used nonsteroidal antiinflammatory drugs or new medications, had not been exposed to intravenous contrast and had no acute illnesses. Given that his creatinine level continued to rise rapidly with no clear etiology, the patient was referred to nephrology.

The patient's family history included autosomal dominant polycystic kidney disease, with 2 first-degree relatives requiring dialysis before age 60 . However, he had undergone radiographic screening with abdominal ultrasonography, which was negative for polycystic kidneys.

At the nephrology clinic, the patient's blood pressure was $149 / 98 \mathrm{~mm} \mathrm{Hg}$, with no urgent clinical indications for dialysis. Renal ultrasonography showed normal-sized kidneys with no hydronephrosis or echogenicity. An incidental $1.2 \mathrm{~cm}$ bladder mass was seen on ultrasonography and was later diagnosed as noninvasive urothelial carcinoma. (This was treated with local excision with interval surveillance, requiring no chemotherapy.)

Urine studies at the patient's initial nephrology visit did not show leukocytes, erythrocytes or protein. There were no cellular casts or crystals seen on urine microscopy. Results of serum and urine protein electrophoresis studies were negative. Complete blood count was normal. However, the patient's serum calcium and parathyroid hormone (PTH) levels showed a non-PTHmediated hypercalcemia (Box 1). Testing of 25-hydroxyvitamin $D_{3}$ and 1,25 dihydroxyvitamin $\mathrm{D}_{3}$ levels was ordered. Imaging studies of the chest and abdomen were unremarkable.

\section{KEY POINTS}

- Vitamin D toxicity is rare, but clinicians must be aware of the risks of vitamin D use to limit complications related to hypercalcemia.

- Calcium levels may get worse before getting better in patients even after cessation of supplements, as vitamin D is fat soluble.

- Observational data and expert opinion suggest that glucocorticoids, ketoconazole and hydroxychloroquine are reasonable options to treat hypercalcemia related to vitamin $D$ toxicity by decreasing the "active" 1,25 dihydroxyvitamin $D_{3}$ levels.

The differential diagnosis at this point included hypercalcemia of malignancy, multiple myeloma and granulomatous diseases, such as sarcoidosis. A renal biopsy showed features of nephrosclerosis and microcalcifications but with no features of sarcoidosis or light chain deposition (Figure 1).

On more detailed questioning, the patient mentioned that he was seeing a naturopathic specialist who had prescribed high doses of vitamin D, advising him to take 8 drops of a specific brand daily. He did not have a history of a fragility fracture or documented vitamin $D$ deficiency. The recommended brand contained 500 IU per drop. Unknowingly, the patient obtained another vitamin D preparation that contained $1000 \mathrm{IU}$ per drop. The patient was not counselled about toxicity risks and, over a period of 2.5 years, he took 8-12 drops of vitamin D daily, for a total daily dose of 8000-12000 IU.

At the nephrology clinic, the patient's measured 1,25 dihydroxyvitamin $\mathrm{D}_{3}$ level was $274 \mathrm{pmol} / \mathrm{L}$ and his 25 -hydroxyvitamin $D_{3}$ level was $241 \mathrm{nmol} / \mathrm{L}$ (Box 1). He was instructed to stop taking all vitamin D supplements and calcium-rich foods. His diuretics remained on hold, but one of his antihypertensive agents (amlodipine) was resumed after his second clinic visit. His 1,25 dihydroxyvitamin $\mathrm{D}_{3}$ and ionized calcium levels continued to increase (Figure 2). His only new symptom related to hypercalcemia was pruritus.

Given his worsening hypercalcemia and increased active vitamin D levels, we counselled the patient about starting glucocorticoid therapy. He was reluctant to start glucocorticoids, given concerns about potential weight gain. As an alternative, we offered oral hydroxychloroquine at $400 \mathrm{mg}$ daily and counselled the patient on adverse effects, including retinal toxicity. 


\begin{tabular}{|c|c|c|c|c|c|c|c|}
\hline Laboratory test & $\begin{array}{l}\text { Visit } \\
\text { no. } 1\end{array}$ & $\begin{array}{l}\text { Visit } \\
\text { no. } 2\end{array}$ & $\begin{array}{l}\text { Visit } \\
\text { no. } 4\end{array}$ & $\begin{array}{l}\text { Visit } \\
\text { no. } 5\end{array}$ & $\begin{array}{l}\text { Visit } \\
\text { no. } 8\end{array}$ & $\begin{array}{l}\text { Visit } \\
\text { no. } 9\end{array}$ & $\begin{array}{l}\text { Normal } \\
\text { range }\end{array}$ \\
\hline lonized calcium, mmol/L† & 1.48 & 1.54 & 1.63 & 1.47 & 1.26 & 1.27 & $1.12-1.32$ \\
\hline Calcium, mmol/L $\ddagger$ & 3.04 & 3.21 & 3.28 & 2.87 & 2.57 & 2.57 & $2.20-2.62$ \\
\hline Phosphate, $\mathrm{mmol} / \mathrm{L}$ & 0.95 & 0.94 & 1.05 & 0.89 & 0.78 & 0.73 & $0.80-1.40$ \\
\hline PTH, ng/L & 4.72 & 2.83 & 3.77 & 10.37 & - & - & $12.26-71.67$ \\
\hline $\begin{array}{l}1,25 \text { dihydroxyvitamin } D_{3} \text {, } \\
\mathrm{pmol} / \mathrm{L} \S\end{array}$ & 274 & 313 & 234 & 187 & 125 & 102 & $60-206$ \\
\hline $\begin{array}{l}\text { 25-hydroxyvitamin } \mathrm{D}_{3} \text {, } \\
\mathrm{nmol} / \mathrm{L} \S\end{array}$ & 241 & 186 & 152 & 135 & 96 & 108 & $25-200$ \\
\hline Creatinine, $\mu \mathrm{mol} / \mathrm{L}$ & 372 & 241 & 271 & 237 & 199 & 186 & $64-110$ \\
\hline $\begin{array}{l}\text { Estimated GFR, mL/ } \\
\mathrm{min} / 1.73 \mathrm{~m}^{2}\end{array}$ & $<15$ & 25 & 22 & 26 & 32 & 34 & $>60$ \\
\hline \multicolumn{8}{|c|}{$\begin{array}{l}\text { Note: GFR = glomerular filtration rate, } \mathrm{PTH}=\text { parathyroid hormone. } \\
\text { *The patient's third clinic visit was a routine visit to check his response to the hydroxychloroquine. Clinic visits occurred every 4-6 weeks. } \\
\text { tCorrected for } \mathrm{pH} 7.4 \text {. } \\
\text { †Corrected for albumin. } \\
\text { §These results became available } 2 \text { weeks after blood was drawn on a clinic visit. }\end{array}$} \\
\hline
\end{tabular}

The patient's calcium and vitamin D levels decreased after initiation of hydroxychloroquine. Almost 1 year after diagnosis, his calcium and vitamin $D$ levels have returned to normal, but he is left with stage $3 B$ (estimated glomerular filtration rate $34 \mathrm{~mL} / \mathrm{min} / 1.73 \mathrm{~m}^{3}$ ) chronic kidney disease.

\section{Discussion}

Historically, reports have outlined the benefits of vitamin $D$ in relation to bone health. ${ }^{1}$ Other purported benefits of vitamin D included nonskeletal outcomes, such as cardiovascular benefit, fall prevention, and reduction of infections and malignancies. ${ }^{2}$ However, an umbrella review of systematic reviews and metaanalyses did not show that vitamin $D$ reduces primary fracture

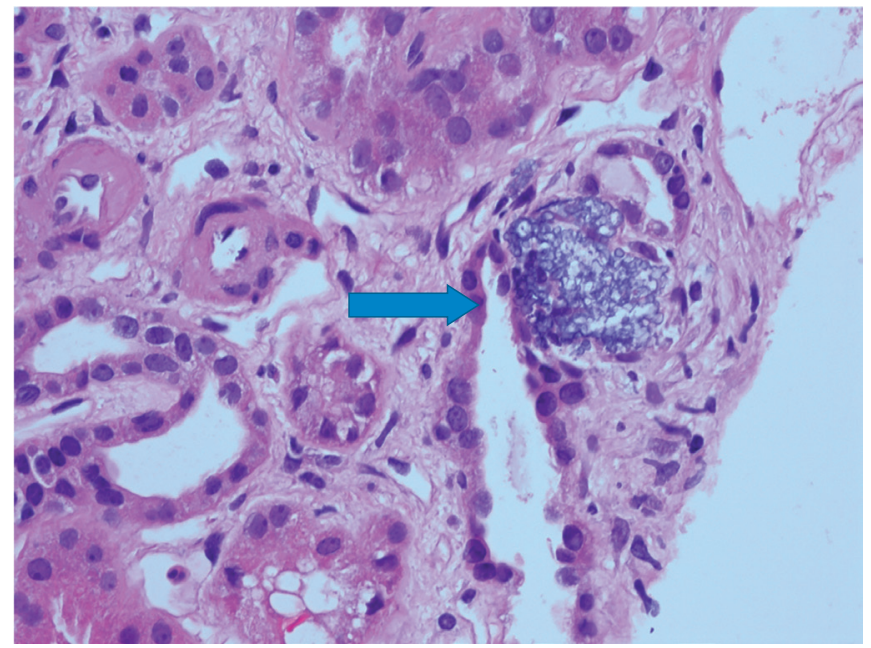

Figure 1: Photomicrograph of the tubulointerstitium of a 54-year-old man with vitamin $D$ toxicity showing microcalcifications (arrow). Arterioles display hyalinosis (hematoxylin-eosin stain $\times 40$ ). risk or convincingly improves other nonskeletal health outcomes. ${ }^{3}$ Furthermore, a recent review for the US Preventive Services Task Force showed no benefit of vitamin $D$ in preventing primary fracture in those without known deficiency, osteoporosis or prior fracture. ${ }^{4}$

In its 2010 guideline, Osteoporosis Canada recommended vitamin D supplementation of $10-25 \mu \mathrm{g}(400-1000$ IU) for most low-risk adults under the age of 50 years to achieve serum levels of 25 -hydroxyvitamin $D_{3}$ greater than $75 \mathrm{nmol} / \mathrm{L}$ (level 3 evidence), ${ }^{1}$ arguing that the potential benefits outweigh risks. A daily vitamin D intake of 20-50 $\mu \mathrm{g}$ (800-2000 IU) is recommended for high-risk and older adults (level 2 evidence). ${ }^{1}$

\section{Mechanism of vitamin $D$ toxicity}

Although vitamin D toxicity is rare owing to a large therapeutic range, its widespread availability in various over-the-counter formulations may pose a substantial risk to uninformed patients. After consumption, vitamin $D$ is carried to the liver where it undergoes hydroxylation and is activated by either microsomal CYP2R1 or mitochondrial CYP27A1 to 25 -hydroxyvitamin $\mathrm{D}_{3}{ }^{5,6}$ The resulting 25 -hydroxyvitamin $D_{3}$ binds to the vitamin $D$ binding protein and is carried to the kidneys for further $1 \alpha$-hydroxylation by CYP27B1 to produce 1,25 dihydroxyvitamin $\mathrm{D}_{3}{ }^{.}$This 1,25 dihydroxyvitamin $D_{3}$ is transported to target cells and enters the nucleus of the vitamin $D$ receptor, leading to an upregulation in gene expression. Although it is transported by vitamin $\mathrm{D}$ binding protein, 1,25 dihydroxyvitamin $\mathrm{D}_{3}$ has a lower affinity to binding relative to 25 -hydroxyvitamin $\mathrm{D}_{3}$ and its metabolites. ${ }^{5} \mathrm{~A}$ leading hypothesis suggests that an oversaturation of the vitamin $D$ binding protein causes an increase in free active Vitamin $D(1,25$ dihydroxyvitamin $D_{3}$ ), resulting in hypercalcemia.

CYP24A1 plays an important role in the deactivation of 1,25 dihydroxyvitamin $D_{3}$ to calcitroic acid. ${ }^{6}$ CYP24A1 also breaks 


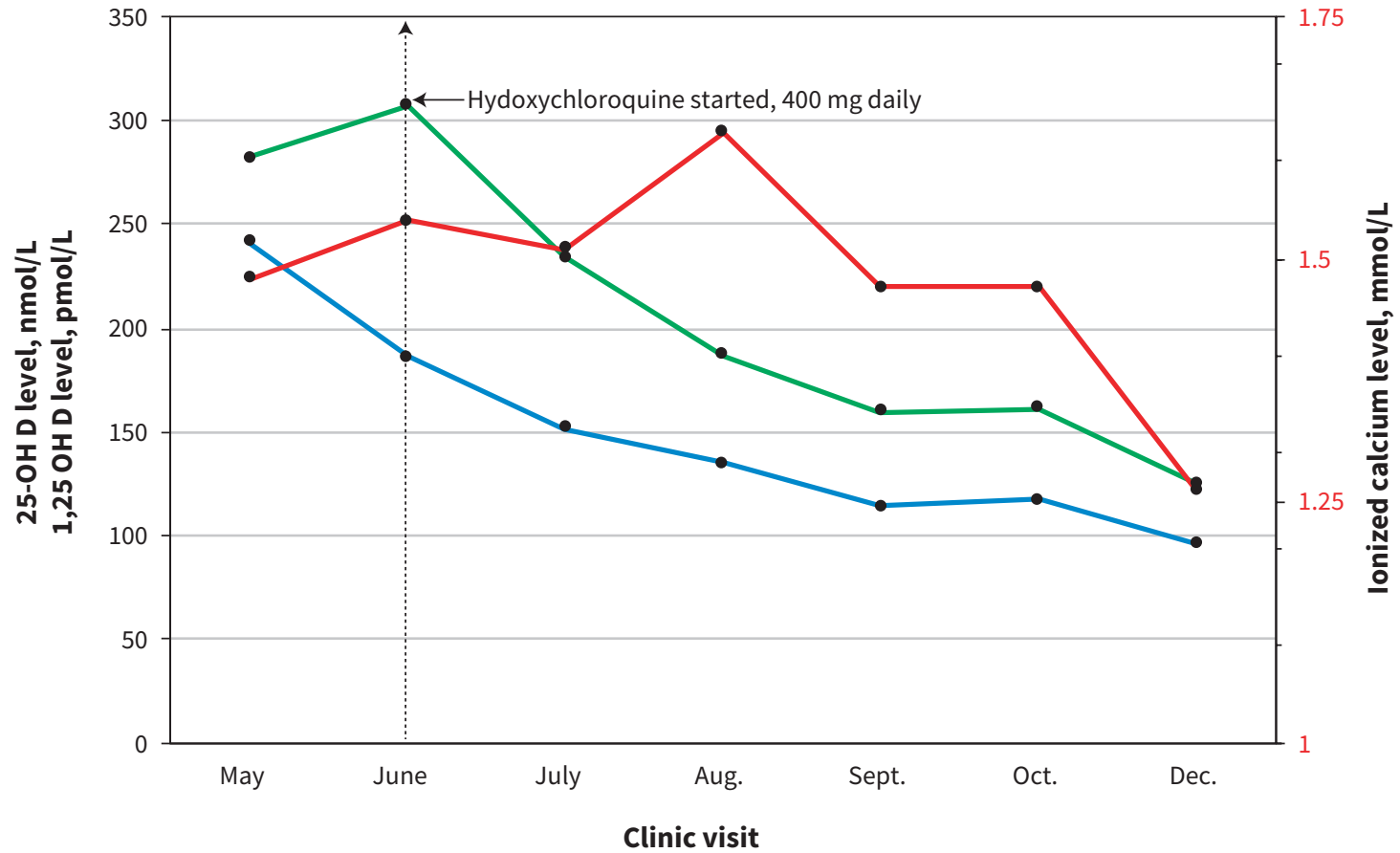

$\bullet 25-\mathrm{OH}$ vitamin D $\longrightarrow 1,25 \mathrm{OH}$ vitamin D $\longrightarrow$ Ionized calcium

Figure 2: Serial 25-hydroxyvitamin $\mathrm{D}_{3}(25-\mathrm{OH} \mathrm{D}), 1,25$ dihydroxyvitamin $\mathrm{D}_{3}(1,25 \mathrm{OH} \mathrm{D})$ and ionized calcium levels over a 10 -month period. Ionized calcium and 1,25 dihydroxyvitamin $\mathrm{D}_{3}$ levels continued to rise 4 weeks after cessation of supplements. Hydroxychloroquine was started at the June clinic visit, and 1,25 dihydroxyvitamin $\mathrm{D}_{3}$ levels steadily decreased after its initiation.

down precursor 25-hydroxyvitamin $\mathrm{D}_{3}$ to 24,25-dihydroxyvitamin $D_{3}$. Loss-of-function mutations in CYP24A1 have been associated with hypercalcemia because of increased vitamin $D$ sensitivity. ${ }^{6}$ Although we did not perform genetic testing on our patient, it is plausible that he had a CYP24A1 mutation, increasing his susceptibility to vitamin $D$ toxicity.

\section{Manifestations of toxicity}

Toxicity can occur over a short period in patients ingesting large doses of vitamin D, either intentionally or inadvertently. ${ }^{7}$ The literature supports that doses greater than $10000 \mathrm{IU}$ per day for several months may lead to toxicity $\left(>200 \mathrm{nmol} / \mathrm{L}\right.$ of 25 -hydroxyvitamin $\left.\mathrm{D}_{3}\right){ }^{8}$ However, differences in patient characteristics such as malabsorption and mutations in CYP24A1 can lead to substantial variation in doses required for toxicity to occur.

Patients may present with symptoms involving the central nervous system and gastrointestinal, genitourinary and cardiovascular systems. Central nervous system manifestations include lethargy, hypotonia, hyporeflexia, confusion and coma. Gastrointestinal symptoms include nausea, vomiting, pancreatitis and constipation; cardiovascular symptoms of toxicity, such as hypertension, arrhythmias and QT segment shortening, may also occur. ${ }^{9}$ Genitourinary symptoms include polyuria, nephrocalcinosis and renal failure. The symptomatology associated with vitamin D toxicity underscores the suggestion that hypercalcemia may be responsible for most symptoms seen. ${ }^{7}$ Sustained hypercalcemia can also lead to dysregulation of calcium-phosphate homeostasis leading to PTH suppression and impaired bone turnover. ${ }^{3,5}$

Importantly, patients may be asymptomatic, delaying diagnosis, and abnormalities related to vitamin D toxicity may be detected only incidentally.

In terms of renal involvement, hypercalcemia can cause kidney injury both acutely and chronically. Hypercalcemia can cause acute kidney injury primarily by 2 mechanisms: afferent arteriolar constriction and intravascular volume depletion from a diuretic effect through activation of calcium-sensing receptor at the sodium-chloride cotransporter in the loop of Henle. ${ }^{10}$ Our patient's acute kidney injury was likely worsened by volume depletion from diuretic use, prolonged heat exposure and preexisting hypercalcemia from vitamin D toxicity. We saw some improvement in his renal function as his calcium levels decreased, by discontinuing his vitamin D supplements and temporarily holding his diuretics and antihypertensive agents. However, we believe that our patient developed chronic disease as shown by nephrosclerosis on renal biopsy (Figure 1).

\section{Management of toxicity}

In cases where vitamin D toxicity is suspected, patients should have their medications - prescribed and over-the-counter carefully reviewed. Initial strategies to reduce vitamin D levels should focus on reducing dietary or supplementary sources. Although there is currently no evidence from large trials, 


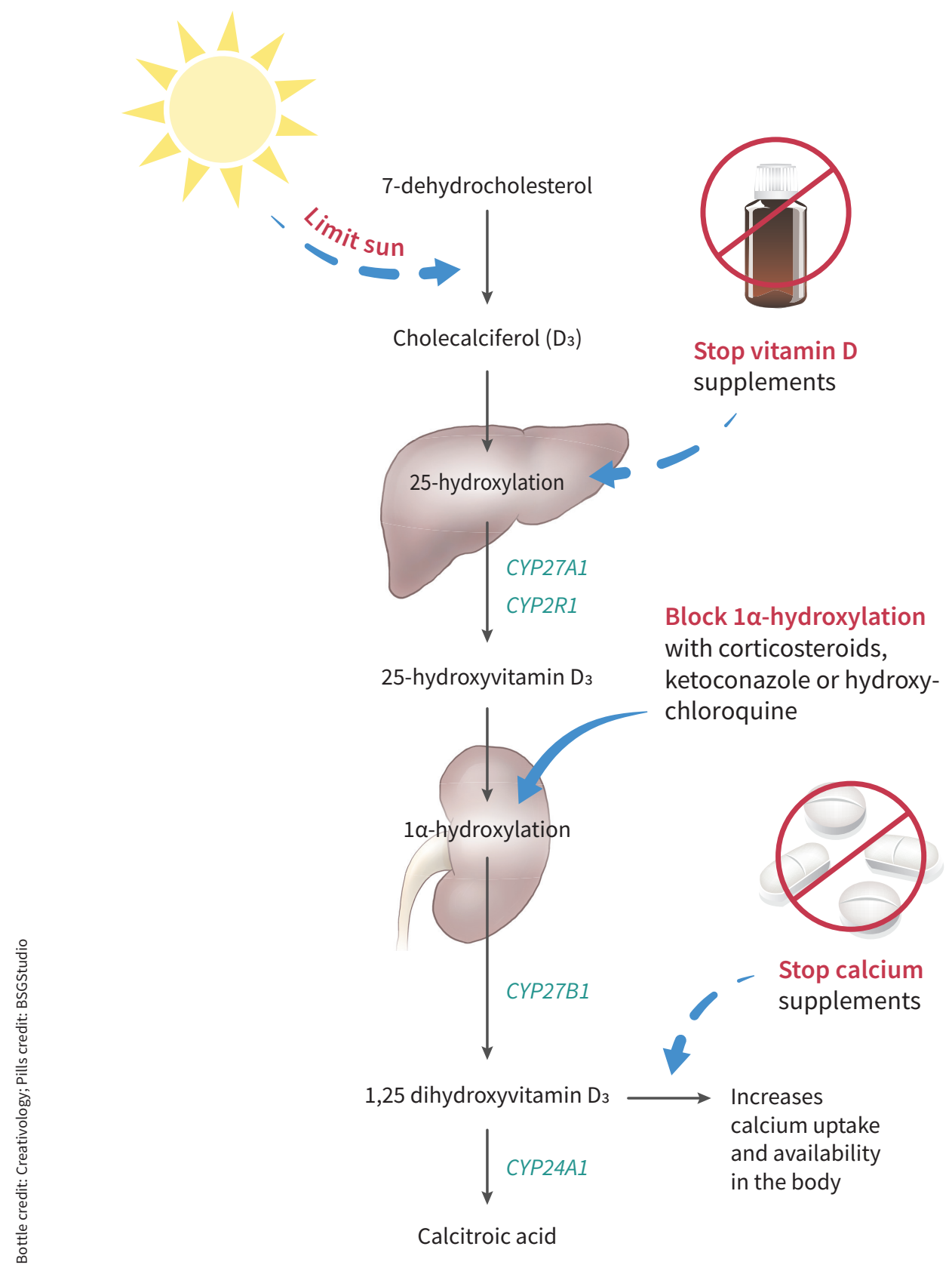

Figure 3: Simplified pathway of vitamin D metabolism with a suggested approach to manage hypervitaminosis D. Key steps include the following: Limit sun exposure; Stop oral vitamin D supplements; Use medications such as corticosteroids, ketoconazole and hydroxychloroquine to block $1 \alpha$-hydroxylase activity; Stop oral calcium supplements to reduce burden of hypercalcemia from hypervitaminosis D. Key enzymes involved in metabolism pathway are shown in green. ${ }^{5-7,11,12}$

observational data suggest that clinicians may consider strategies to reduce active vitamin $D$ levels if hypercalcemia persists, through inhibition of $1 \alpha$-hydroxylase activity (Figure 3)..$^{11,12}$ If patients are asymptomatic, clinicians may choose to monitor levels expectantly, as vitamin $D$ is very fat soluble and levels may take some time to return to normal.

Several medications have been used successfully to treat hypercalcemia by reducing the active form of vitamin D. Glucocorticoids, ketoconazole and hydroxychloroquine have all been used in cases of hypercalcemia related to sarcoidosis. ${ }^{1,9}$ Expert opinion suggests that these medications reduce $1 \alpha$-hydroxylase activity and may be used to manage hypercalcemia by reducing 1,25 dihydroxyvitamin $D_{3}$ levels. ${ }^{11,12}$ Given that our patient was reluctant to use glucocorticoid treatment, we used hydroxychloroquine as an alternative to decrease his 1,25 dihydroxyvitamin $\mathrm{D}_{3}$ levels and, in turn, his calcium levels.

Our experience informs us that patients and clinicians should be better informed about the risks regarding the unfettered use 
of vitamin D. Given new findings from the US Preventive Services Task Force, ${ }^{4}$ current Canadian guidelines regarding its use in lowrisk individuals should be revisited.

Patients with CYP24A1 mutations may be at an increased risk of vitamin D toxicity, and clinicians can consider genetic testing if vitamin D toxicity develops with doses less than $10000 \mathrm{IU}$ per day. Although vitamin $D$ toxicity is rare, early recognition may prevent chronic complications related to hypercalcemia. In patients who are symptomatic, cessation of supplements along with treatment with glucocorticoids is suggested. In cases where glucocorticoid therapy is not preferred or is contraindicated, ketoconazole or hydroxychloroquine are reasonable alternatives.

\section{References}

1. Hanley DA, Cranney A, Jones G, et al.; Guidelines Committee of the Scientific Advisory Council of Osteoporosis Canada. Vitamin D in adult health and disease: a review and guideline statement from Osteoporosis Canada (summary). CMAJ 2010;182:1315-9.

2. Bischoff-Ferrari HA, Dawson-Hughes B, Staehelin HB, et al. Fall prevention with supplemental and active forms of vitamin D: a meta-analysis of randomised controlled trials. BMJ 2009;339:b3692.

3. Theodoratou E, Tzoulaki I, Zgaga L, et al. Vitamin D and multiple health outcomes: umbrella review of systematic reviews and meta-analyses of observational studies and randomised trials. BMJ 2014;348:g2035.

4. Kahwati LC, Weber RP, Pan H, et al. Vitamin D, calcium, or combined supplementation for the primary prevention of fractures in community-dwelling adults: evidence report and systematic review for the US Preventive Services Task Force. JAMA 2018;319:1600-12.

5. Jones G. Pharmacokinetics of vitamin D toxicity. Am J Clin Nutr 2008;88:582S-6S.

6. Schlingmann KP, Kaufmann M, Weber S, et al. Mutations in CYP24A1 and idiopathic infantile hypercalcemia. N Engl J Med 2011;365:410-21.

7. Koul PA, Ahmad SH, Ahmad F, et al. Vitamin d toxicity in adults: a case series from an area with endemic hypovitaminosis d. Oman Med J 2011;26:201-4.

8. Hathcock JN, Shao A, Vieth R, et al. Risk assessment for vitamin D. Am J Clin Nutr 2007;85:6-18.
9. Kaptein S, Risselada AJ, Boerma EC, et al. Life-threatening complications of vitamin D intoxication due to over-the-counter supplements. Clin Toxicol (Phila) 2010;48:460-2.

10. Riccardi D, Brown EM. Physiology and pathophysiology of the calcium-sensing receptor in the kidney. Am J Physiol Renal Physiol 2010;298:F485-99.

11. Sharma OP. Vitamin D, calcium, and sarcoidosis. Chest 1996;109:535-9.

12. Barré PE, Gascon-Barré M, Meakins JL, et al. Hydroxychloroquine treatment of hypercalcemia in a patient with sarcoidosis undergoing hemodialysis. Am J Med 1987;82:1259-62.

Competing interests: None declared.

This article has been peer reviewed.

The authors have obtained patient consent.

Affiliations: Division of Nephrology (Auguste, Bargman), University of Toronto; Department of Pathology (Avila-Casado), Toronto General Hospital, University Health Network, Toronto, Ont.

Contributors: All authors participated in the conception, design, writing and revision of the article. All authors approved the final version of the manuscript to be published and agree to be accountable for all aspects of this work.

Correspondence to: Bourne Auguste, bourne.auguste@mail. utoronto.ca

The section Cases presents brief case reports that convey clear, practical lessons. Preference is given to common presentations of important rare conditions, and important unusual presentations of common problems. Articles start with a case presentation (500 words maximum), and a discussion of the underlying condition follows (1000 words maximum). Visual elements (e.g., tables of the differential diagnosis, clinical features or diagnostic approach) are encouraged. Consent from patients for publication of their story is a necessity. See information for authors at www.cmaj.ca. 